\title{
The Diagnostic and Prognostic Role of Vascular Endothelial Growth Factor C in Sepsis and Septic Shock
}

\author{
Emoke Almasy ${ }^{1}$, Janos Szederjesi ${ }^{1}$, Bianca Liana Grigorescu² ${ }^{*}$, Iudita Badea ${ }^{1}$, \\ Marius Petrisor ${ }^{3}$, Cristina Manasturean ${ }^{4}$, Valentina Negrea ${ }^{4}$, Agota-Evelyn Timar ${ }^{1}$, \\ Oana Coman ${ }^{1}$, Leonard Azamfirei ${ }^{1}$, Ario Santini ${ }^{5}$, Sanda Maria Copotoiu ${ }^{1}$ \\ ${ }_{1}$ Anesthesiology and Intensive Care Clinic, George Emil Palade University of Medicine, Pharmacy, Science, and \\ Technology of Targu Mures, Romania \\ 2 Discipline of Pathophysiology, George Emil Palade University of Medicine, Pharmacy, Science, and Technology of \\ Targu Mures, Romania \\ 3 Department of Simulation Applied to Medicine, George Emil Palade University of Medicine, Pharmacy, Science, and \\ Technology of Targu Mures, Romania \\ 4 Infectious Diseases Clinic, University Hospital Targu Mures, Romania \\ 5 The University of Edinburgh, UK
}

\begin{abstract}
Introduction: Variations in the expression of vascular endothelial growth factor (VEGF) could be used as a biomarker in critically ill patients with sepsis and septic shock. Inflammation potently upregulates VEGF-C expression via macrophages with an unpredictable response. This study aimed to assess one of the newer biomarkers (VEGF-C) in patients with sepsis or septic shock and its clinical value as a diagnostic and prognostic tool. Material and methods: The study involved 142 persons divided into three groups. Group A consisted of fifty-eight patients with sepsis; Group B consisted of forty-nine patients diagnosed as having septic shock according to the Sepsis -3 criteria. A control group of thirty-five healthy volunteers comprised Group C. Severity scores, prognostic score and organ dysfunction score, were recorded at the time of enrolment in the study. The analysis included specificity and sensitivity of plasma VEGF-C for diagnosis of septic shock. Circulating plasma VEGF-C levels were correlated with the APACHE II, MODS and severity scores and mortality. Results: The mean (SD) plasma VEGF-C levels in septic shock patients (1374(789) pg./m), on vasopressors at the time of admission to the ICU, were significantly higher $1374(789) \mathrm{pg} . / \mathrm{mL}$, compared the mean (SD) plasma VEGF-C levels in sepsis patients (934(468) pg./mL); $(p=0.0005$, Student's t-test.) Plasma VEGF$C$ levels in groups A and B were shown to be significantly correlated with the APACHE II $(r=0.21, p=0.02 ; r=0.45, p$ $=0.0009)$ and MODS score $(r=0.29, p=0.03 ; r=0.4, p=0.003)$. There was no association between plasma VEGF-C levels and mortality $[p=0.1]$. The cut-off value for septic shock was $1010 \mathrm{pg} . / \mathrm{ml}$. Conclusions: VEGF-C may be used as a prognostic marker in sepsis and septic shock due to its correlation with APACHE II values and as an early marker to determine the likelihood of developing MODS. It could be used as an early biomarker for diagnosing patients with septic shock.
\end{abstract}

Keywords: vascular endothelial growth factor C, sepsis, septic shock, lymphangiogenesis

Received: 20 August 2019 / Accepted: 23 April 2020

\section{INTRODUCTION}

Due to the life-threatening potential of sepsis, early diagnosis and treatment are the keys in the successful management of the illness. This is the reason why, in the last years, a panel of new biomarkers have been developed, to facilitate a better approach in sepsis management $[1,2]$. Some of these markers are important for diagnosis and prognosis, likewise with respect to their genetic variability $[3,4]$.

$\mathrm{C}$ reactive protein (CRP), procalcitonin $(\mathrm{PCT})$ and pro-inflammatory cytokines (interleukin six and interleukin eight) are biomarkers routinely utilized in the diagnosis and prognostic stratification [3]. Although the biomarkers mentioned above have an important di- 
agnostic and prognostic role, their specificity is low and have not been proved to have an appreciable impact on patient outcome or mortality [5]. As such, identification of a biomarker with a strong early predictive value in sepsis would have significant therapeutic value in managing patients with sepsis and improving their clinical evolution.

The current literature supports the hypothesis that vascular endothelial growth factor (VEGF) could be used as a prognostic marker in critically ill patients with sepsis and septic shock. Leung (1989) described VEGF as a factor related to hypoxic stress [6].

Sepsis and septic shock are two pathological conditions arising due to an exaggerated immune response to pathogens. Inflammation impairs tissue perfusion secondary to micro-thrombosis, vascular leakage leading to oedema, increased interstitial fluid pressure, and compression of vessels resulting in impaired tissue oxygenation [7]. Hypoxia-inducible factor (HIF) plays a significant role in the transactivation of target genes such as VEGF [7]. Nitric oxide and cytokines influence VEGF expression, thus, elevated circulating concentrations of VEGF were noticed in sepsis, inducing lymphatic endothelial cell proliferation and survival $[7,8]$.

Three major classes of VEGF receptors are expressed in vascular and extravascular tissues [7]. All family members of VEGF receptors are tyrosine kinase receptors.

Previous studies have suggested that VEGF receptor-1 and VEGF receptor-2 on vascular endothelial cells mediate angiogenesis, whereas VEGF-3 regulates lymphangiogenesis $[8,9,10]$. Type C VEGF belongs to an angiogenic regulator family, alongside VEGF-A, VEGF-B, VEGF-D and placental growth factor (PIGF) $[8,10]$.

VEGF-A is commonly known for its involvement in blood vessel morphogenesis. Conversely, VEGF-C interacts predominantly with VEGF receptor 3 (VEGFR-3) on lymphatic endothelial cells and is responsible for lymphangiogenesis $[8,10]$.

This study aimed to assess plasma VEGF-C levels as a diagnostic and prognostic tool for sepsis and septic shock states.

The Null hypotheses are:

1.There is no difference between plasma vascular endothelial growth factor- C (VEGF-C) levels in the sepsis and control groups.
2. There is no difference between plasma vascular endothelial growth factor- C (VEGF-C) levels in the septic shock and control groups.

3. There is no difference between plasma vascular endothelial growth factor- C (VEGF-C) levels in the sepsis and septic shock groups.

4. There is no difference in endothelial growth factor- C (VEGF-C) levels between the sepsis and septic shock groups in relationship to qSOFA, SOFA scores or APACHE II and MODS, scores.

\section{MATERIAL AND METHODS}

The study was conducted from Feb 1, 2018, to Jul 30 2018, in the Intensive Care Unit of the County Emergency Clinical Hospital, Targu Mures, Romania.

Written informed consent was obtained before the patients being enrolled in the study. The same procedure was applied to healthy volunteers included as controls.

The regional ethics committees approval (no 19248/2017) was obtained.

For patients unable to give consent, their legal representatives gave consent on their behalf.

One hundred and forty-two patients were enrolled and divided into three groups. Group A consisted of fifty-eight patients with sepsis, Group B comprised of forty-nine patients with septic shock, diagnosed according to the Sepsis - 3 criteria. Group C (Controls) consisted of thirty-five healthy volunteers.

All patients in Groups A and B were enrolled on the day when sepsis or septic shock onset was first diagnosed and recorded.

Inclusion criteria: Adult patients aged 18-80 years, with sepsis or septic shock. In accordance to the Sepsis-3 definition, diagnosis of sepsis and septic shock was determined by clinical criteria of infection using the Quick Sequential Organ Failure Assessment (qSOFA) score $\geq 2$, and a Sequential Organ Failure Assessment (SOFA) score $\geq 2$ [2].

Exclusion criteria were: Known malignancy or neoplasm, history of recent surgery, hematologic disorders, cardiopulmonary resuscitation and patients with autoimmune diseases that could cause expression of high plasma VEGF-C levels.

Once enrolled, a medical history was taken, and the patient underwent a clinical examination. 
The obtained data and parameters were recorded as well as the vasoactive treatment and dosage, before enrolment. Regardless of whether vasopressor therapy was initiated before ICU admission or during ICU stay, plasma samples for VEGF-C levels were obtained after vasopressor treatment.

The length of stay in the ICU was recorded either at time of death or on discharge from the unit.

Acute Physiology and Chronic Health Evaluation (APACHE II) prognostic score and Multiple Organ Dysfunction Syndrome (MODS) scores were recorded during the first twenty-four hours after sepsis or septic shock onset and diagnosis.

Blood cultures and bacteriological determinations were performed on the day of enrolment to confirm the diagnosis. The SOFA and qSOFA severity scores were recorded.

\section{Analysis of the samples}

Blood samples to determine plasma VEGF-C levels were collected via vein puncture from the antecubital fossa and were collected in ethylenediaminetetraacetic acid (EDTA) plasma tubes. The samples were centrifuged within thirty minutes of collection and stored at $-30^{\circ} \mathrm{C}$ before processing. Enzyme-Linked Immuno Sorbent Assay (ELISA) test (R\&D Systems, Minneapolis, USA) was used and were processed with the Dynex DSX ELISA System in the Centre for Advanced Medical and Pharmaceutical Research, Targu Mures.

The sensitivity of the VEGF-C analysis kit was 13.3 pg./ml.

Routine blood tests were performed daily. Due to the lack of laboratory reagents, CRP and PCT were not assessed in every patient.

Plasma lactate levels were assessed daily using an arterial blood gas analyzer (Stat Profile Prime Plus by Nova Biomedical UK).

Statistical analysis was performed using Microsoft Excel [Microsoft, Washington, USA], Graphpad Prism [Graphpad Software, Inc., California, USA] and the Statistical Package for Social Sciences (SPSS version 17, Chicago, IL, USA].

All quantitative variables were tested for normality using the D'Agostino test. Non-Gaussian data were analyzed using the Mann-Whitney non-parametric test.

For normal distributed data, the Student's t-test and ANOVA analysis were used.
Correlations were tested using Spearman test, for non-Gaussian distributed data.

Chi-squared test and Fisher's exact test were used for comparing the distribution of nominal values. Correlations were tested using Spearman test, for non-Gaussian distributed data. In order to assess the influence of noradrenaline dose and mean arterial pressure value on the determined plasma VEGF-C level, a linear regression model, using VEGF-C level as the dependent variable was constructed.

The analysis included specificity and sensitivity of plasma VEGF-C levels in patients diagnosed with septic shock.

Graphic representation of receiver-operating-characteristic (ROC) was used, with the determination of area under the curve (AUC).

The significance level was set at $\alpha=0.05$.

\section{DESULTS}

The demographic data and length of stay in ICU for Group A (sepsis) and Group B (septic shock) are given in Table 1 . Age and gender were not significantly different between Groups A and B. ( $p>0.05$, Student's t-test).

Differences between septic and septic shock patients regarding their severity scores are shown in Table 2. There were significant differences between the two

Table 1. Demographic data and length of stay in ICU for Group A (sepsis) and Group B (septic shock)

\begin{tabular}{lccc} 
Variables & Group A & Group B & P-value \\
\hline $\begin{array}{l}\text { Age(years) } \\
\text { median (range) }\end{array}$ & $65(68)$ & $69(56)$ & $0.17^{* *}$ \\
Gender, male (n, \%) & $35(60 \%)$ & $22(44 \%)$ & \\
Gender, female (n, \%) & $23(40 \%)$ & $27(56 \%)$ & $0.12^{*}$ \\
\hline "Chi-square test; " Mann-Whitney test & & &
\end{tabular}

Table 2. qSOFA, SOFA, MODS, APACHE II scores in Groups $A$ and $B$

$\begin{array}{lccc}\text { Variables } & \text { Group A } & \text { Group B } & \text { P value } \\ \begin{array}{l}\text { qSOFA score (median) } \\ \text { SOFA score }\end{array} & 2 & 2 & >0.999 \\ \text { (median, range) } & 5(0-16) & 8(0-18) & <0.0001 \\ \begin{array}{l}\text { MODS score } \\ \text { (median, range) }\end{array} & 4.9(0-10) & 9.2(2-18) & <0.0001 \\ \begin{array}{l}\text { APACHE II score } \\ \text { (median, range) }\end{array} & 17(6-39) & 23(5-39) & <0.0001\end{array}$

"Mann-Whitney test; "* Fisher's exact test; QSOFA Quick Sequential Organ Failure Assessment; SOFA Sequential Organ Failure Assessment; MODS Multiple Organ Dysfunction Syndrome; APACHE Il Acute Physiology and Chronic Health Evaluation. 
groups with regard to SOFA, APACHE 11 and MODS, $(\mathrm{p}<0.001)$ but not qSOFA. $(\mathrm{p}>0.999)$.

No statistically significant difference was observed between plasma VEGF-C levels in Group A (sepsis) and Group C (controls) ( $\mathrm{p}=0.9$, Student's test). Null hypothesis 1 . is therefore upheld.

The mean (SD) of plasma VEGF-C levels in Group $B$ (septic shock) was significantly higher than plasma VEGF-C levels in Group C (controls) ( $\mathrm{p}=0.001$, Student's t-test). Null hypothesis 2 . is therefore rejected.

The mean (SD) of plasma VEGF-C levels in Group A (sepsis) 934(468) pg./mL were significantly lower than Group B (septic shock) 1374(789) ( $\mathrm{p}=0.0005$, Student's t-test).

Null hypothesis 3. is therefore rejected.

There was a significant difference between the plasma VEGF-C levels in Group A \& Group B in respect to SOFA, MODS and APACE II scores ( $p<0.001$ in all three cases, Mann-Whitney test) but not to qSOFA scores. $(\mathrm{p}>0.999$, Mann-Whitney test) Null hypothesis 4 . is therefore partially rejected.
There was no significant correlation between plasma VEGF-C levels in group A and SOFA $(r=0.14$, $p$ $=0.12)$ or $\mathrm{qSOFA}(\mathrm{r}=0.11, \mathrm{p}=0.2)$, respectively between plasma VEGF-C levels in group $\mathrm{B}$ and SOFA ( $\mathrm{r}$ $=0.11, \mathrm{p}=0.4)$ or $\mathrm{qSOFA}(\mathrm{r}=0.17, \mathrm{p}=0.2)$ severity scores. [Spearman rank test]. Plasma VEGF-C levels in groups $\mathrm{A}$ and $\mathrm{B}$ were shown to be significantly correlated with the APACHE II ( $\mathrm{r}=0.21, \mathrm{p}=0.02 ; \mathrm{r}=0.45$, $\mathrm{p}=0.0009)$ and MODS score $(\mathrm{r}=0.29, \mathrm{p}=0.03 ; \mathrm{r}=0.4$, $\mathrm{p}=0.003)$. The statistical power for these correlations with APACHE II are 0.63 and 0.92 , respectively 0.58 and 0.84 with MODS scores.

No significant correlation between plasma VEGF-C levels and plasma lactate level was found in groups $\mathrm{A}$ and $B(r=0.08 p=0.3 ; r=0.05, p=0.6)$. [Spearman rank test]

Multivariate analysis revealed a positive significant correlation between plasma VEGF-C levels, and vasoactive medication dose for Group B (septic shock) ( $\mathrm{r}=$ $0.975, \mathrm{p}=<0.0001$, Student's $\mathrm{t}$-test)

The mean arterial pressure and mortality were both significantly higher in Group B (septic shock) than in

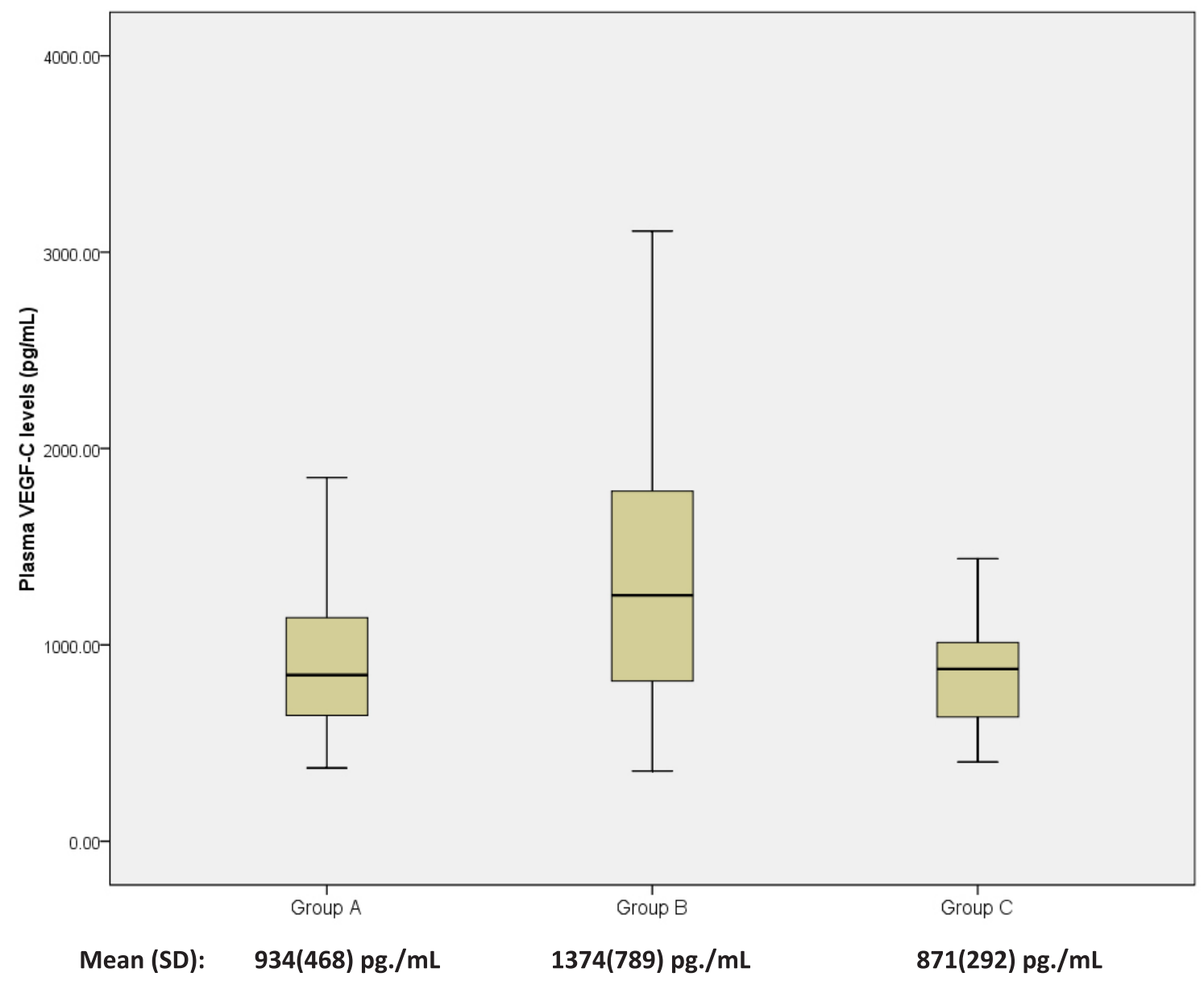

Fig. 1. Mean values of plasma VEGF-C levels in Group A (sepsis), Group B (septic shock) and Group C (healthy controls) 
Group A (Sepsis) ( $p=0.0002$, Mann-Whitney test).

The length of stay in the ICU was significantly higher in patients in Group A (Sepsis) than in Group B (septic shock). ( $\mathrm{p}=0.0002$, Mann-Whitney test)

No significant correlation with plasma lactate level was found ( $\mathrm{p}=0.3$. test)

There was a significant difference between the mean (SD) values of plasma VEGF-C levels from the sepsis group (Group A), the septic shock group (Group B) and the controls (Group C). ( $\mathrm{p}=0.0001$, ANOVA) . An F coefficient of 10.73 was obtained. The maximum plasma VEGF-C levels for all three groups were 3268 pg./mL, $3988 \mathrm{pg} . / \mathrm{mL}$, and $1439 \mathrm{pg} / \mathrm{mL}$, respectively. The horizontal bolded line on the box-plots represents the median value, the $50^{\text {th }}$ percentile. The upper limit of the box-plot represents the $75^{\text {th }}$ percentile, and the inferior limit of the box-plot represents the $25^{\text {th }}$ percentile. The horizontal lines that extend out of the box-plots represent the maximal and minimal plasma VEGF-C level in that group that are not extremes or outliers.

Figure 2 indicates that the plasma VEGF-C level was $71.43 \%$ for specificity and $61.22 \%$ for sensitivity; both "specific" and "sensitive" for the diagnosis of septic shock.

The cut-off plasma VEGF-C level for diagnosis of septic shock was established at $1010 \mathrm{pg} / \mathrm{mL}$.

In order to assess the influence of noradrenaline and mean arterial pressure over plasma VEGF-C levels, a linear regression model was constructed.

A significant influence of noradrenaline and mean arterial pressure over plasma VEGF-C levels ( $\mathrm{R}$ square $=0.601$, Beta $=0.787,0.130)$ was observed.

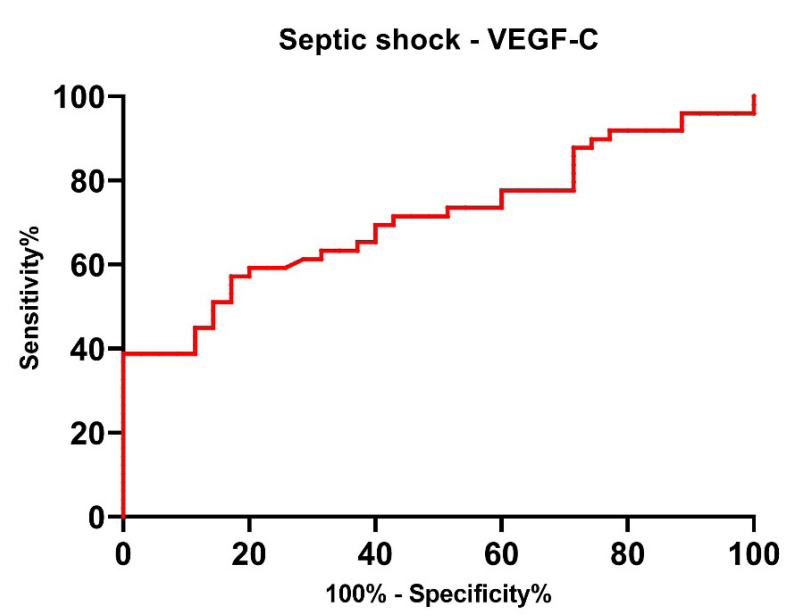

Fig. 2. ROC curve for plasma VEGF-C levels for septic shock (AUC $=0.709, p=0.001$ )
The analysis showed no correlation between measured plasma VEGF-C levels in Groups A \& B and hospital mortality $(\mathrm{p}=0.1)$.

In the two subgroups, there was a significantly higher in-hospital mortality rate in Group B. $(\mathrm{p}=<0.0001)$. [Fisher's exact test]

\section{DISCUSSION}

Sepsis is a pathological condition that can occur in critically ill patients admitted to an intensive care unit regardless of the underlying condition. The onset, course and development of septic shock or MODS are related to the virulence of the pathogen(s), to the comorbidities of the patient and their response to infection $[1,2]$.

Regarding the life-threatening potential of sepsis and septic shock, the development of newer and faster diagnostic biomarkers is mandatory.

Despite years of research, morbidity and mortality from sepsis remain unacceptably high [11].

The medical literature indicates a role of vascular endothelial growth factor in the systemic inflammatory response syndrome, a common feature of critically ill patients regardless of the underlying pathology, and in sepsis $[10,12,13]$.

Consecutively, endothelial dysfunction can be influenced by the drugs that septic patients receive during therapy, not only directed related to sepsis, but also with other associated dysfunctions: anticoagulants, vasoactive, antihypertensives, calcium channel blockers, so endothelial alteration should be evaluated not only in the context of the underlying disease but as well as for the applied therapy $[14,15]$. Equally important is not only the proper administration but also the biological properties, pharmacokinetics and plasma levels of each of these drugs $[16,17]$.

The diagnostic and prognostic value of $C$ type VEGF in sepsis, which promotes lymphangiogenesis, remains unclear, and its role in this requires further investigation.

In our study, no correlation between plasma VEGF$\mathrm{C}$ levels and the patients' age was observed in agreement with other published studies $[13,18]$.

Significantly higher values of circulating plasma VEGF-C levels were found in the group with sepsis than in the non-infected healthy controls, regardless of the demographic data. This observation is in agreement with Zhang et al. (2014), who provided evidence 
that plasma VEGF-C level is increased in patients with sepsis [10].

An important finding in the present study was that higher plasma VEGF-C levels were found in patients in the septic shock group who were undergoing vasoactive treatment. Only two other reports have described a significantly increased plasma VEGF-C level during septic shock $[10,18]$.

Patients who had increased plasma VEGF-C levels needed increased amounts of vasoactive medication.

A positive significant correlation between plasma VEGF-C levels and vasoactive medication doses were noticed. The severe hypotension occurring in septic shock is related to large amounts of bradykinin and histamine release by a systemic inflammatory response to a bacterial injury, then an aggressive vasoactive medication was required.

Histamine and VEGF induced vascular leakage leads to plasma exudation with increased interstitial fluid pressure, decreased peripheral blood flow and impaired tissue oxygenation.

The hypoxic injury will stimulate an inflammatory response, which induces VEGF-C expression [7]. All patients enrolled in the study received oxygen therapy. In Group B (septic shock), the early introduction of vasopressors diminished hypoxia at tissue level, and plasma lactate levels did not vary significantly from baseline levels. This may explain why a statistically significant correlation between plasma VEGF-C levels and plasma lactate levels was not obtained.

In the current study, it was noticed that increased amounts of noradrenaline and the mean arterial pressure values influence plasma VEGF-C levels in $60 \%$ of cases.

There is a vicious circle maintaining the inflammatory response and leading to multisystem organ failure.

The septic shock group had higher mortality rates compared to the group with sepsis; however, we were unable to find a significant correlation between plasma VEGF-C levels and mortality [18].

Above the established calculated cut-off value, the likelihood ratio of developing septic shock was two times higher. Even if increased plasma VEGF-C levels were found in septic shock patients, VEGF-C cannot be considered as a specific marker due to its relatively low sensitivity and specificity. Therefore, its utility in diagnosing septic shock is limited.
Above a level of $1055 \mathrm{pg} / \mathrm{mL}$, the likelihood occurrence of septic shock was three times higher, with a specificity of $82 \%$. However, in this case, the sensitivity was found to be low at $57,14 \%$.

Several other studies have reported a decrease in circulating VEGF level for non-survivors. Two studies indicated an increase in C-type VEGF levels in patients who subsequently died. These results are not consistent with our study, which reveals that increased plasma VEGF-C levels were not related to mortality $[13,18]$.

In the septic shock group, there were fewer ventilator-free days and length of stay in ICU. These findings may be attributed to high mortality rates and a shorter duration of survival.

Related to prognostic scores, Shapiro et al. (2008) concluded that a positive correlation exists between VEGF and APACHE II scores [19]. Our study also supports this finding.

There is a lack of evidence regarding the correlation between plasma VEGF-C levels, APACHE II and MODS scores in the literature. The present study showed a positive correlation between these variables.

No significant correlation was found between plasma VEGF-C levels and SOFA severity score. This finding can be explained by the fact that critically ill patients with sepsis did not possess high SOFA scores secondary to sepsis alone, but rather as a result of chronic renal or hepatic pathologies, chronic obstructive bronchopulmonary disease or heart failure [20].

Zhang et al. (2014) suggested that the vascular endothelial growth factor receptor-3 (VEGFR-3) and its ligand VEGF-C are protective during sepsis by attenuating pro-inflammatory processes [21].

The inflammation process effectively upregulates VEGF-C expression via macrophages, promoting resolution in some cases or exacerbating response in others.

Evolution may be unpredictable depending on the patient's immune status and degree of hypoxia, which, if too severe, will impair VEGF-C production [6].

\section{- CONCLUSIONS}

VEGF-C may be used as a prognostic marker in sepsis and septic shock due to its correlation with APACHE II values and as an early marker to determine the likelihood of developing MODS. It could be used as an early biomarker for diagnosing patients with septic shock. 


\section{- CONFLICT OF INTEREST}

None to declare.

\section{REFERENCES}

1. Managing Sepsis and Septic Shock: Current Guidelines and Definitions. AJN 2018; 118(2):34-39.

2. Singer $\mathrm{M}$, Deutschman $\mathrm{CS}$, Seymour $\mathrm{CW}$ et al. The third international consensus definitions for sepsis and septic shock (Sepsis-3). JAMA 2016; 315(8): 801-10.

3. Georgescu AM, Grigorescu BL, Chirteș IR, Vitin A, Fodor RS. The Relevance of Coding Gene Polymorphisms of Cytokines and Cellular Receptors in Sepsis. The Journal of Critical Care Medicine 2017;3(1):5-11.

4. Georgescu AM, Banescu C, Badea I et al. IL-6 gene polymorphisms and sepsis in ICU adult romanian patients: a prospective study. Rev Romana Med Lab. 2017; 25(1):75-89.

5. Nargis W, Ibrahim MD, Ahamed BU. Procalcitonin versus C-reactive protein: Usefulness as biomarker of sepsis in ICU patient. Int J Crit IIIn Inj Sci. 2014 Jul-Sep; 4(3): 195-199.

6. Rauniyar K, Kumar Jha S, Jeltsch M. Biology of Vascular Endothelial Growth Factor $C$ in the Morphogenesis of Lymphatic Vessels. Front Bioeng Biotechnol. 2018; 6: 7.

7. S. Ramakrishnan, Anand V, Roy S.. Vascular Endothelial growth factor signalling in hypoxia and Inflammation. J Neuroimmune Pharmacol. 2014 Mar; 9(2): 142-160.

8. Adams RH, Alitalo K. Molecular regulation of angiogenesis and lymphangiogenesis. Nat Rev Mol Cell Biol. 8(6): 464-478.

9. Azamfirei L, Gurzu S, Solomon R et al. Vascular endothelial growth factor: a possible mediator of endothelial activation in acute respiratory distress syndrome. Minerva Anestesiologica. 2010 August;76(8):609-16.

10. Y. Zhang, Lu $Y, M a L$ et al. Activation of vascular endothelial growth factor receptor-3 in macrophages restrains TLR4-NF$\mathrm{KB}$ signalling and protects against endotoxin shock. Immunity. 2014; 40:501-514.
11. Schorr C, Zanotti S, Dellinger RP. Severe sepsis and septic shock. Virulence. 2014 Jan 1; 5(1): 190-199.

12. Yano K, Liaw PC, Mullington JM et al. Vascular endothelial growth factor is an important determinant of sepsis morbidity and mortality. J Exp Med. 2006 Jun 12; 203(6): 1447-1458.

13. Karlsson S, Pettila V, Tenhunen J et al. Vascular Endothelial Growth Factor in Severe Sepsis and Septic Shock. Anesth Analg, 2008;106:1820-1826.

14. Tilea I, Popa DS, Xantus TSZ et al. Determination of Apixaban Levels in Human Plasma by a High-Throughput Liquid Chromatographic Tandem Mass Spectrometry Assay. Rev Romana Med Lab. 2015; 23(1):115-126.

15. Namba S, Yamaoka-Tojo M, Kakizaki $R$ et al. Efects on bone metabolism markers and arterial stifness by switching to rivaroxaban from warfarin in patients with atrial fibrillation. Heart Vessels. 2017; 32(8):977-982.

16. Varga A, Farczadi L, Vlase L et al. Liquid Chromatography Tandem Mass Spectrometry Simultaneous Determination of Amlodipine and Telmisartan in Human Plasma for Therapeutic Drug Monitoring. Rev Chim. 2015; 66(10):1675-1679.

17. Di Filippo L, Dos Santos KC, Hanck-Silva G et al. A Critical Review of Biological Properties, Delivery Systems and Analytical/ Bioanalytical Methods for Determination of Bevacizumab. Crit Rev Anal Them. 2020; DOI: 10.1080/10408347.2020.1743641.

18. EL-Akabawy H, Hamela MA, Gaber A, Abozekry A. Prognostic value of vascular endothelial growth factor in sepsis syndrome. The Egyptian Journal of Critical Care Medicine 2016; 4:119126.

19. Shapiro IN, Yano K, Okada H et al. A prospective, observational study of soluble FLT-1 and vascular endothelial growth factor in sepsis. Shock. 2008 Apr; 29(4): 452-457.

20. Raith EP, Udy AA, Bailey $M$ et al. Prognostic accuracy of the SOFA score, SIRS criteria, and qSOFA score for in-hospital mortality among adults with suspected infection admitted to the intensive care unit. JAMA 2017; 317(3): 290-300.

21. Zhang RY, Zhang H, Huang J, Qu HP, Tang YQ. Angiogenic factors in sepsis: are we ready for the new therapeutic era? Crit Care. 2014; 18(1): 403. 